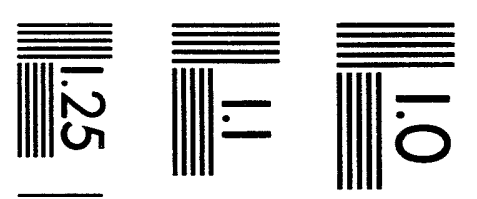

$$
\begin{aligned}
& \text { 福 }
\end{aligned}
$$

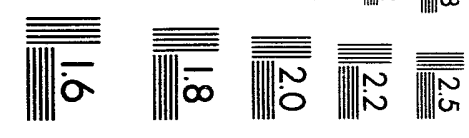



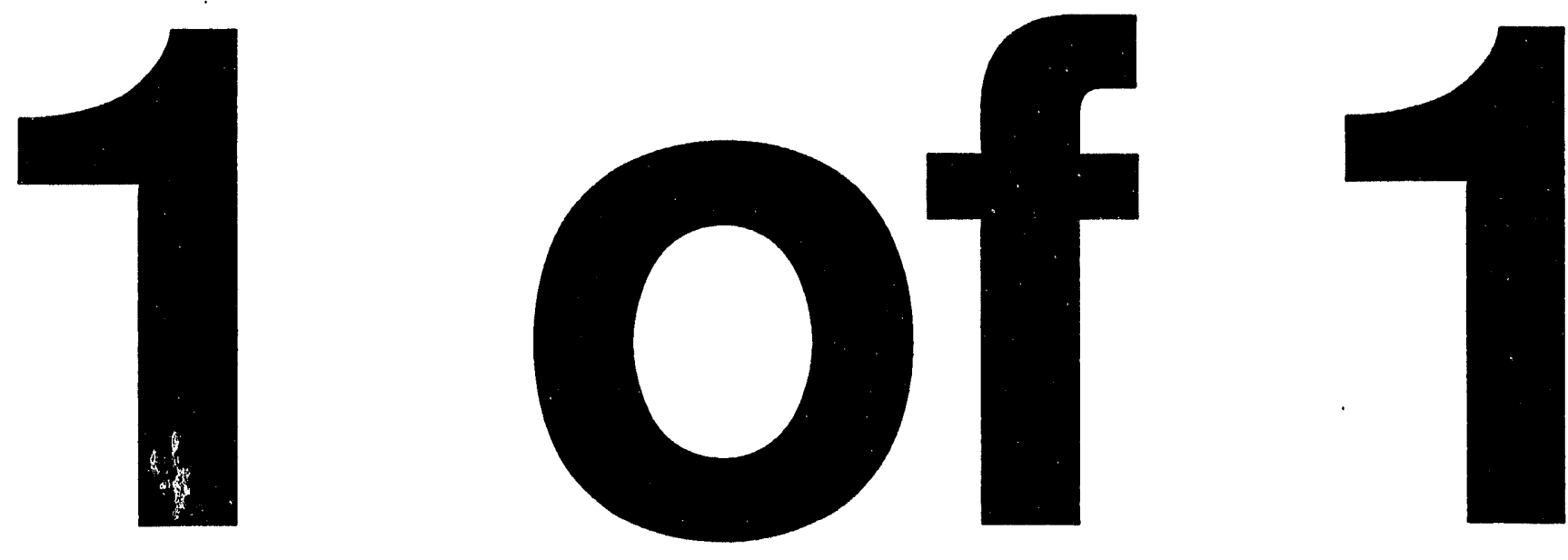
LA-UR- 93-3599
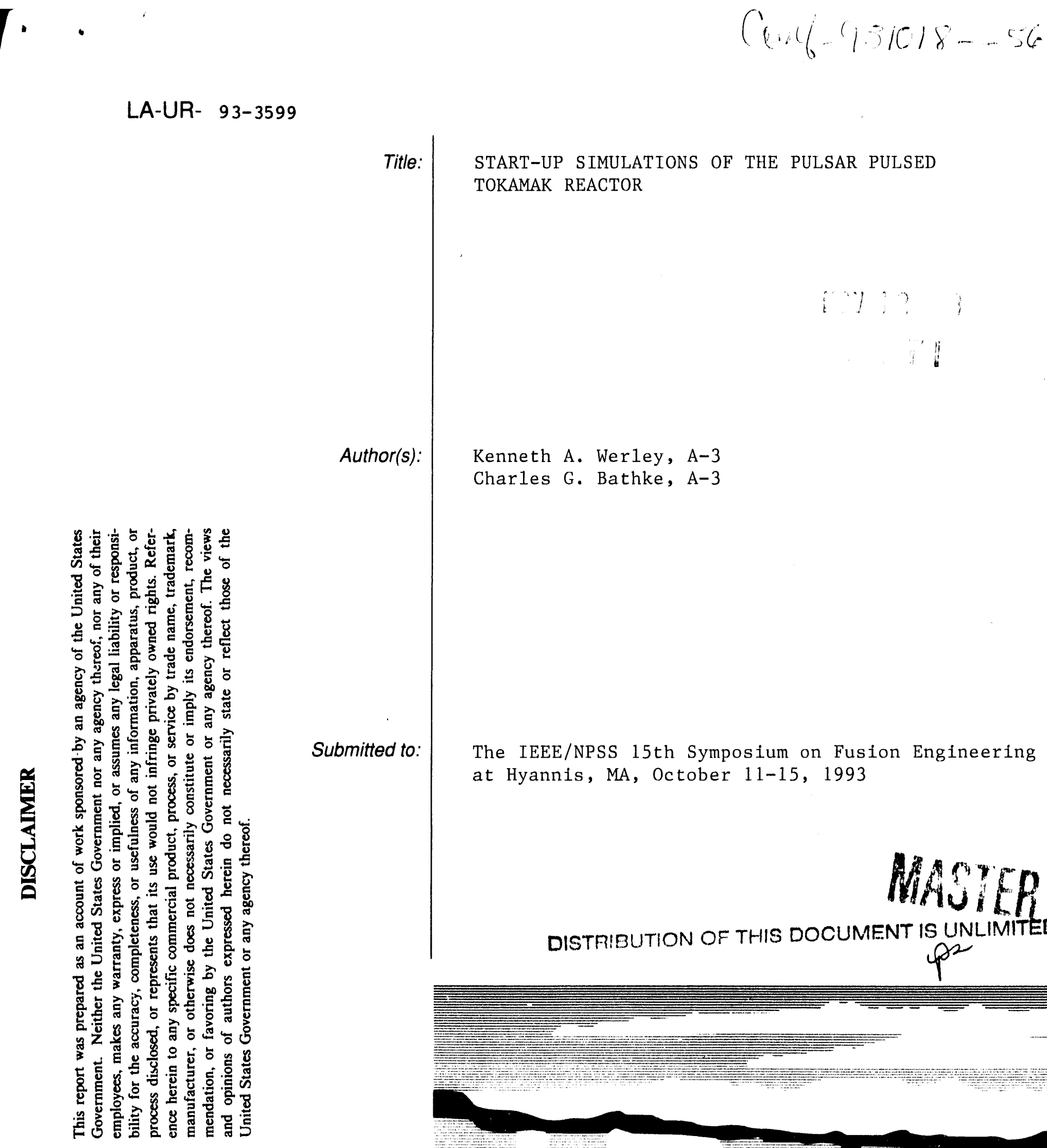

START-UP SIMULATIONS OF THE PULSAR PULSED TOKAMAK REACTOR

Kenneth A. Werley, A-3

Charles G. Bathke, A-3

Submitted to:

The IEEE/NPSS 15th Symposium on Fusion Engineering at Hyannis, MA, October 11-15, 1993

\section{Los Alamos} WATIONAL LABOAATOOY 


\title{
Start-Up Simulations of the PULSAR Pulsed Tokamak Reactor ${ }^{\dagger}$
}

\author{
K.A. Werley and C.G. Bathke \\ Los Alamos National Laboratory, P.O. Box 1663, Los Alamos, NM 87545, USA
}

\begin{abstract}
Start-up conditions are examined for a pulsed tokamak reactor that uses ouly inductively driven plasma current (and bootstrap current). A zero-dimensional (profile-averaged) model containing plasma power and particle balance equations is used to study several aspects of plasma start-up, including: (1) optimization of the start-up pathway; (2) tradeoffs of auxiliary start-up heating power versus start-up time; (3) volt-second consumtion; (4) thermal stability of the operating point; (5) estimates of the divertor heat flux and temperature during the start-up transient; (6) the sensitivity of the available operating space to allowed values of the $H$ confinement factor; and (7) partial-power operations.
\end{abstract}

\section{INTRODUCTION}

Following the Advanced Reactor Innovation and Evaluation Study (ARIES [1-3]) of steady-state tokamaks is the PULsed Advanced Reactor (PULSAR [4]), which features pulsed operation using only inductive current drive. The plasma start-up path and time are especially important to pulsed reactors for which short down times between power pulses reduce energy storage requirements. Fig. 1 illustrates a time history of a single PULSAR pulse for the plasma current and fusion power. The present study examines the current risetime, $\tau_{R}$, and the plasma ignition time, $\tau_{I}$, in order to define an optimum start-up pathway that for a given auxiliary heating power will have the shortest start-up time. This start-up time versus heating power trade-off information is given to the PULSAR systems code (ASC [1]) for determining cost-optimized reactor designs.[4] Additional start-up issues include the volt-seconds consumed, the divertor loadings, disruption and electron-run-away density limits, and operation at partial power levels for meeting reactor plant liscencing and load-following requirements. Several steady-state operating point issues are also examined including the thermal stability and the sensitivity of the available operating space to the choice of the $\mathbf{H}$ confinement factor. The models are applied to a PULSAR design with a plasma current of $I_{\phi}=11.5 \mathrm{MA}$, a major radius of $R_{T}=7.4 \mathrm{~m}$, a minor radius of $a=1.9 \mathrm{~m}$, and an average net electric power of $1 G W e$. This represents an interim PULSAR design point which will evolve as systems model refinements are applied.

†This work was supported by the U.S. Department of Energy under Contract No. W-7405-ENG-36

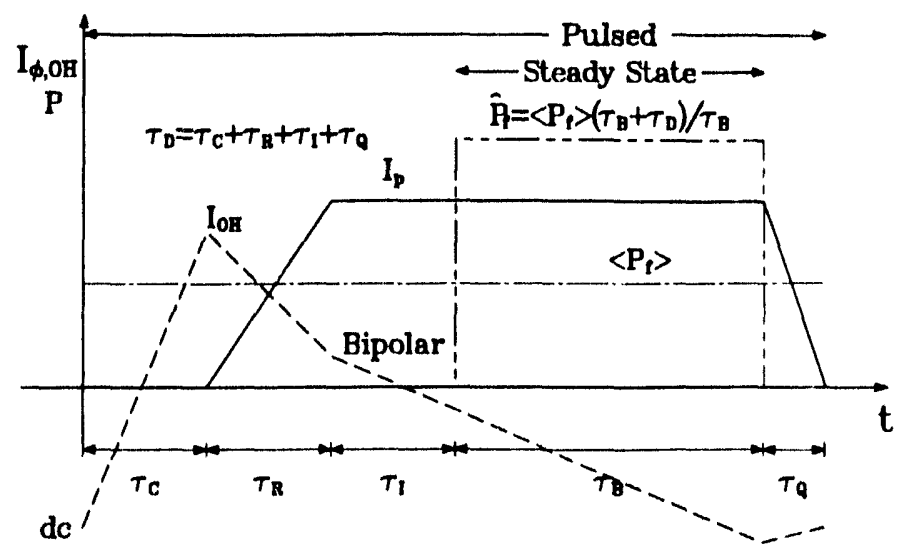

Fig. 1. A schematic of a PULSAR single-pulse time history. The

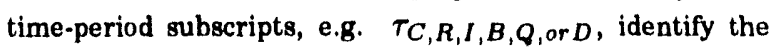
coil Charge (C), plasma current Rise (R), Ignition (I), plasma Burn (B), plasma current Quench (Q), and the total Downtime between burns (D).

\section{ZERO-DIMENSIONAL PIAASMA MODELS}

The plasma model includes ion and electron energy balance; protium, deuterium, tritium, helium-3 and helium-4 (alpha) particle continuity; a specified ionic fraction for a high- $Z$ impurity; charge balance; and magnetic equilibrium constraints. The $0-D$ equations are obtained through a radial average over specified plasma profiles. A detailed description of the model equations can be found in [5] and [1].

The ion and electron energy-balance equations include fusion, auxiliary and ohmic heating, electron-ion equipartition, transport, bremmstrahlung, cyclotron and line radiation. The fractional fusion power deposited in the ions is calculated through a time integral over the slowing down time of the slowing down rate on the ions; zero prompt loss is assumed. Cyclotron radiation loss is a function of the effective wall reflectivity, $R_{C Y C}$, which includes the effects of wall reflection and holes in the wall. The fractional auxiliary power going to the ions is $f_{A U X i}=0.05$, and the axiliary power, $P_{A U X}$, must be specified.

All background ions are assumed to share the same local temperature, $T_{i}(r)$. The total ion density, n, the electron density, $n_{e}$, (assuming charge neutrality) and the effective ionic charge, $Z_{\text {eff }}$, are obtained by summing over the ion species. The average $Z$ and $Z^{2}$ 
of impurity species and line radiation are calculated based upon the assumption of coronal equilibrium. All ion species are assumed to have the same particle confinement time, $\tau_{p}$. The fractional composition of the refuel, as well as the ratio of $\tau_{p} / \tau_{E}=4$, must be specified. The transport energy losses are represented in terms of the ion and electron transport energyconfinement times, which are assumed to be equal. The energy confinement time is given by the ITER-89p [6] expression multiplied by $H=2.2$. The fractional beta carried by the athermal (hot) fusion products, $f_{\beta H}$, is calculated by the slowing down model and is used in computing the plasma beta, $\beta$. The operatingpoint beta is constrained through the specification of a Troyon [5] constant, $C_{T}$, such that $\beta=C_{T} I_{\phi} / a B$. The equilibrium is set as in the systems code [4] through the specification of $I_{\phi} / a B$, elongation, $\kappa=$ 1.812 , triangularity, $\delta=0.705$, and the inverse aspect ratio, $\epsilon$. To complete the $0-\mathrm{D}$ description, plasma profiles for density, temperature, and current density are specified, and profile form factors are calculated for all terms.

This plasma model is incorporated into four numerical codes used for different purposes. Three of the codes are restricted to steady-state conditions. The MakNTAU code applies no transport sraling relation, but rather constrains $\beta$ through a specified Troyon coefficient, $C_{T}=0.032$. This code is used to study the required $n \tau_{E} v s . T_{i}$ space, and also is used within the ASC systems code. The second code, MakTiN, applies a confinement scaling relation rather than a beia constraint, and is used for calculating plasma operating points and their thermal stability. The MakPOP code is similar to MakTiN except that all operating space is surveyed and contours of constant $P_{A U X}$ are plotted for given $I_{\phi}, a, B$ and $\epsilon$.

The time-dependent plasma physics model, BURN, is similar to that described above with the main addition being that a Fokker-Planck formalism is used to describe the time-dependent slowing down of the charged-particle fusion products. The plasma current is constrained to rise exponentially to the steady-state value. The current risetime is determined by systems code cost optimizations. The bootstrap fraction is calculated using the ASC steady-state model that has been corrected by a multiplicative factor consisting of the ratio of the resistive to the total toroidal voltage, where the total voltage includes both resistive and inductive terms. This factor corrects for timedependent effects. The auxiliary heating power is separated into two phases. During the current ramp, $P_{A U X}$ is adjusted to maintain $\epsilon \beta_{\theta}$ at 0.45 , subject a maximum $P_{A U X}$ constraint. Once the plasma has ignited (crossed the saddle point), $P_{A U X}$ is reduced to zero.
One concern during startup is that plasma-wall interactions may be a source of impurities that will enter and degrade the main plasma. To monitor the potential for sputtering erosion, the analytic divertor model of Cooke $[1,8]$, which was developed for highrecycle reactor divertor conditions, is used. This model estimates the peak divertor-plate heat flux and the peak plasma temperature. The model has been benchmarked against two-dimensional edge-transport codes for the ARIES I operating-point. The divertor model is not expected to describe early startup accurately, and is used only to gauge the relative loading compared to a steady-state high-recycle divertor.

The plasma initial conditions used in the simulation are $I_{\phi}(0)=0.25 \mathrm{MA}$ and $T_{e}(0)=T_{i}(0)=0.4 \mathrm{keV}$. The initial plasma density is constrained so that the initial streaming parameter, which is the ratio of the electron drift speed to the thermal speed, has a value of 0.2 Above this value, the electric fields would generate significant numbers of run-away electrons.

Summarizing the models, the plasma continuity equations, together with the profile form factors and the magnetic equilibrium constraints, form a complete set of equations for calculating plasma conditions. Input parameters include $I_{\phi}, a$, the magnetic topology, $\tau_{p} / \tau_{E}$, profile exponents, high-Z impurity fractions, particle fueling fractions, and auxiliary heating information, $P_{A U X}$ and $f_{A U X i}$. The models are used to calculate $n \tau_{E}$, plasma operating points and heating contours, and start-up trajectories.

\section{RESULTS}

The PULSAR-9 (case 9 ) required $n_{i} \tau_{E}$ and $H$ space is shown in Fig. 2. The PULSAR-9 basecase has a $10 \mathrm{keV}$ ion temperature, which is near the minimum $\mathrm{H}$ value. The sensitivity of power balance to the assumed $\mathrm{H}$ factor is illustrated in Fig. 3. As $\mathrm{H}$ becomes smaller than the assumed operating point value of 2.2 , the power-balance space is very sensitive to $H$ and shrinks rapidly. The tritium burn-up fraction is 0.1 , and is about half of a typical ARIES value.

Fig. 4 contains plasma operating cotntours of the auxiliary heating power, and also shows an optimized start-up path that traverses through the ignition boundary saddle point using a maximum auxiliary power of 42MW. The minimum power to cross the saddle point is about $20 \mathrm{MW}$, however, for a maximum $P_{A U X}$ of 21,42 or $63 \mathrm{MW}$, the respective ignition times, $\tau_{I}$, are 153, 59 and $45 \mathrm{~s}$. The ignition time is defined as the time from the beginning of current flattop until $95 \%$ of full power is achieved. Optimal start-up features a short start-up time in order to minimize the downtime between fusion power generation, and low auxiliary 


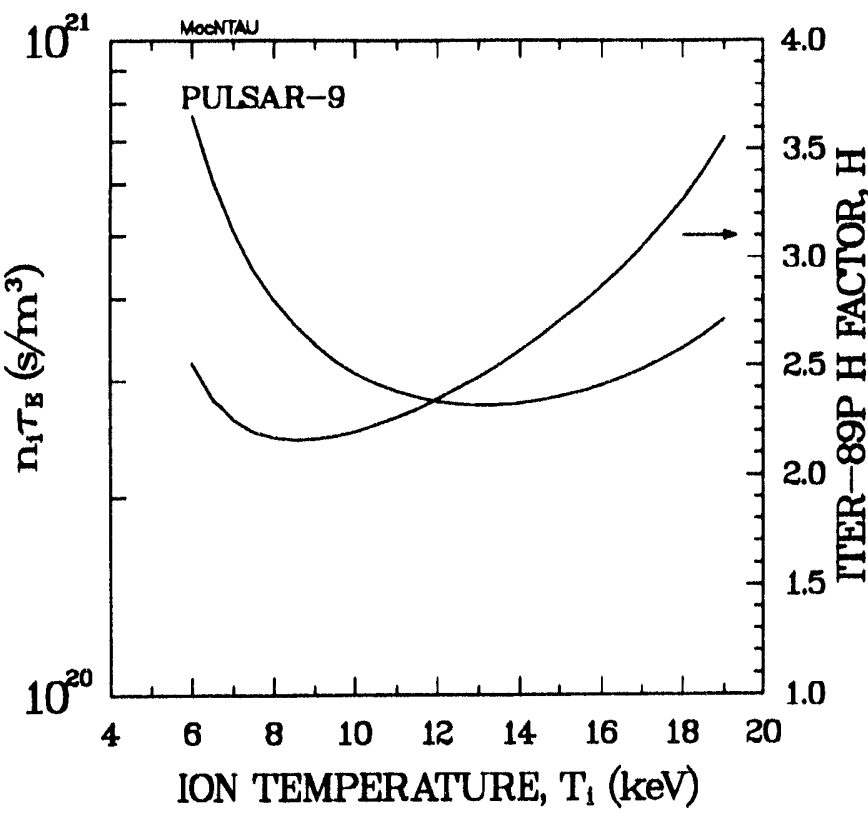

Fig. 2. The required $n_{i} \tau_{E}$ and $H$ confinement parameter versus ion temperature for a Troyon coefficient of $C_{T}=$ $0.032 T \mathrm{~m} / M A$, and for given $I_{\phi}, a, B$ and $\epsilon$.

heating power to minimize heating costs. In practice, a cost-optimized trade-off between the start-up time and $P_{A U X}$ is determined using a systems code.

The time hiscories of the plasma current, temperature and density are shown in Fig. 5. The plasma current is taken to full current to provide the best confinement prior to raising the density and the fusion power to cross over the saddle and achieve ignition. Fig. 6 shows plasma powers during start-up for a maximum $P_{A U X}=42 M W$. A $15 \%$ overpower occurs above th:? operating point condition. This overpower event can be reduced by lowering $P_{A U X}$ at the price of increasing the start-up time. The electron streaming parameter has an initial value of 0.2 and decreases throughout start-up, so electron run-away production would not be a problem for normal operation. The predicted divertor heat load is significantly less than the operating-point value throughout start-up until the ignition overpower event when a small $15 \%$ increase occurs. The predicted peak divertor plasma temperature is significantly higher than the operating value only for about the first $10 \mathrm{~s}$ of startup, when particle fluxes will be low. This predicted high edge temperature $(0.2 \mathrm{kev})$ could be reduced by careful density control at early times without increasing taul. The plasma density is held to about 2/3 of the Greenwald density limit [8] for major plasma disruptions during most of the start-up. During the final stages $n_{e}$ is increased to the operating value $150 \%$ of the Greenwald limit. The ITER CDA design [6] allowed $125 \%$ and the ARIES designs [1-3] permitted $200-400 \%$ of the Greenwald density limit.

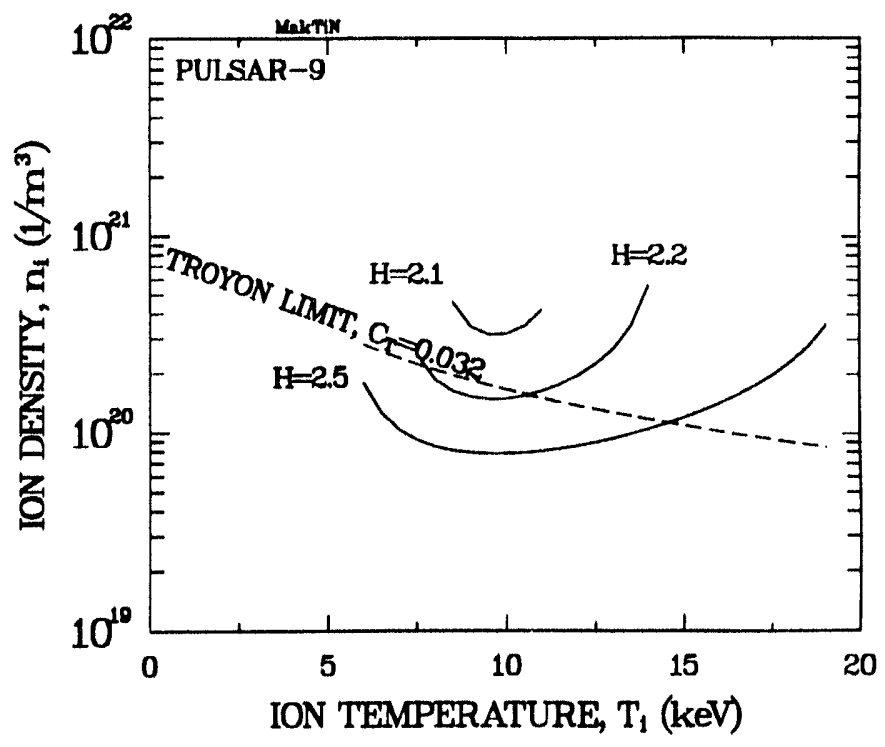

Fig. 3. Ion density versus temperature plasma power balance conditions assuming ITER-89p confinement scaling and a specifled H-confinement factor.

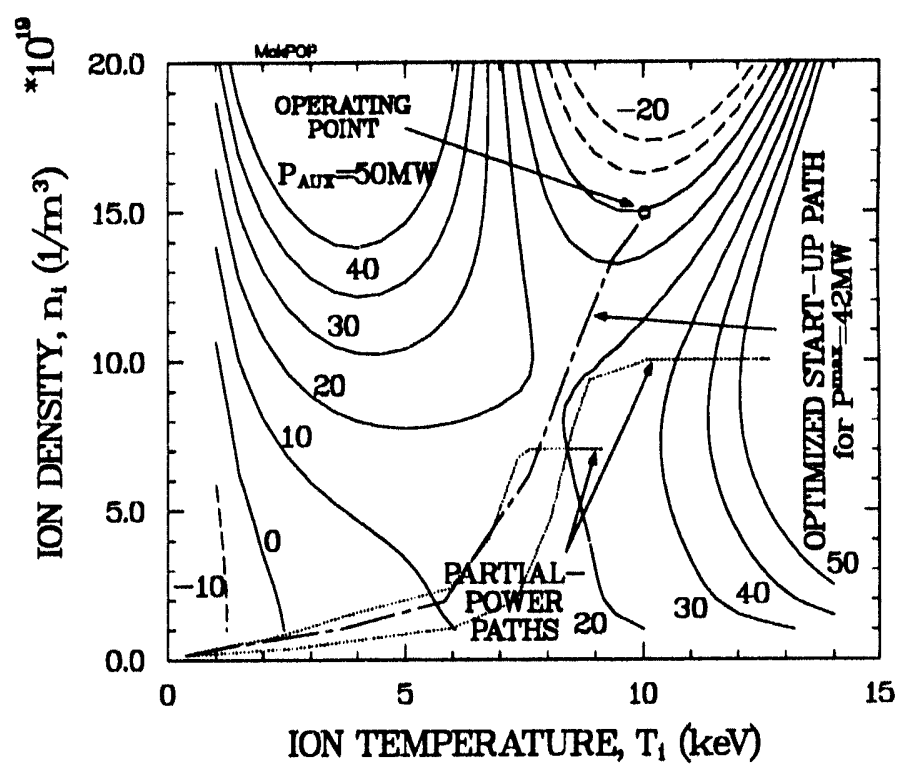

Fig. 4. Plasma operating contours of the auxiliary heating power for PULSAR-9 assuming ITER-89p confinement scaling and an H-confinement factor of 2.2 .

The auxiliary heating contours of Fig. 4 suggest that thermally stable partial-power operation can be achieved anywhere along the start-up path. To demonstrate this stability, the BURN code was used to increase the density during start-up to a low-value cap of $10^{20} \mathrm{~m}^{-3}$ and to maintain $42 \mathrm{MW}$ of auxiliary heating, and a lower $n_{i}=0.7 \times 10^{20} \mathrm{~m}^{-3}$ and $P_{A U X}=21 \mathrm{MW}$. The resulting paths and thermally stable operating points are shown in Fig. 4 by dotted lines. These cases achieve $60 \%$ and $25 \%$ partial powers. 


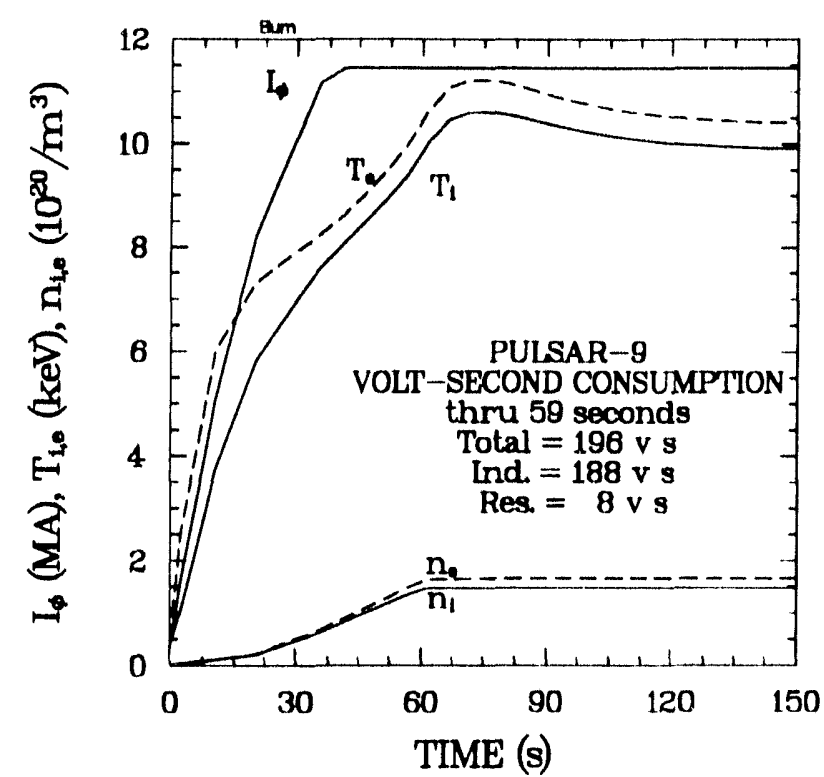

Fig. 5. The plasma current, density-weighted average temperature and average density temporal history during start-up.

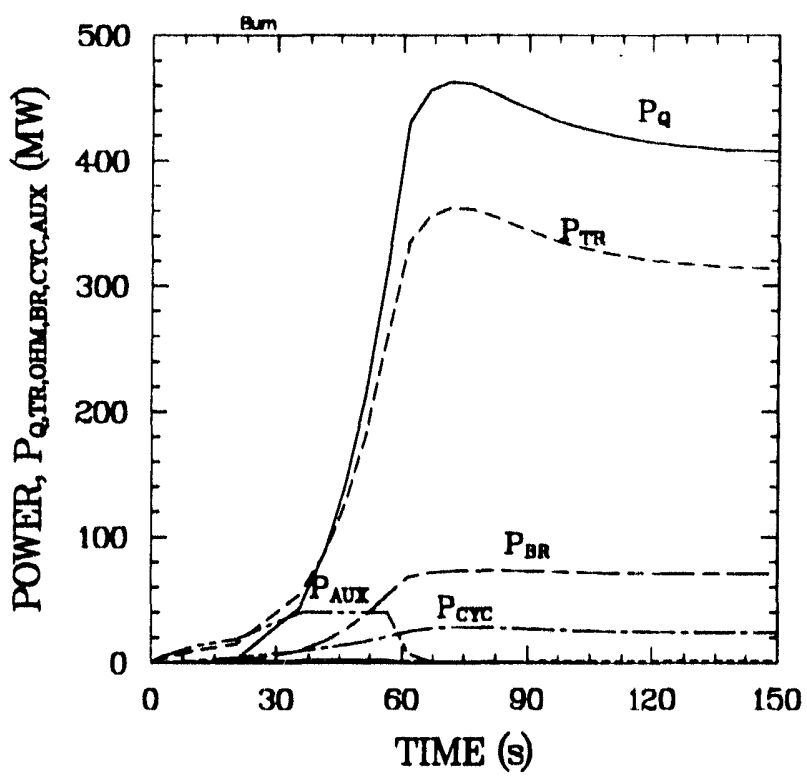

Fig. 6. The plasma power temporal history, included are the fusion plasma heating, $P_{Q}$, the non-radiative transport loss, $P_{T R}$, the bremmstrahlung and cyclotron radiative loss, $P_{B R}$ and $P_{C Y C}$, and the auxiliary heating, $P_{A U X}$.

\section{SUMMARY}

The inductive start-up scenario achieves ignition with no significant electron-runaway problems, with relatively low wall/divertor loading requirements compared to the operating point, and with densities below disruption limits until the operating point conditions are approached. The start-up volt-second consumption is reasonable at $196 \mathrm{Vs}$, with only an $8 \mathrm{Vs}$ resistive loss.
The PULSAR-9 start-up features only a $15 \%$ overpower event during ignition. This percentage could be reduced, but only at the expense of a longer start-up time. The operating point is thermally stable, requires $\mathrm{H}=2.2$, and has a tritium burn-up fraction of 0.1 . Significant cost penalties result for lower $\mathrm{H}$ values.

The start-up pathway is chosen so that partial-power operation is attainable through control of plasma fueling and auxiliary heating. Thermally stable operation at any partial-power level can be achieved for $P_{A U X}$ between $20-30 \mathrm{MW}$. These steady partialpower plasma conditions would feature high plasma temperatures (above $8 \mathrm{keV}$ ) and low plasma resistance. Partial-power operations are necessary for liscensing and/or load following requirements.

In conclusion, plasma start-up of an inductively driven PULSAR reactor is relatively straightforward compared to the start-up of the RF-driven, strongly controlled plasma profiles, and (possibly) secondstability regime requirements of the steady-state ARIES designs. Since the start-up scenario occurs frequently for a pulsed reactor, it plays a strong role in determining a cost-optimized design. In particular, a balance between the start-up time and the startup auxiliary heating power directly affects the reactor energy-storage requirements and the optimal pulse length. The plasma models described herein calculate the optimal start-up path and provide $\tau_{I}$ versus $P_{A U X}$ information to reactor systems-code optimizer routines. The plasma models also are used to characterize thermal stability, partial-power operation, Hfactor confinement requirements, and various start-up parameters of interest.

\section{REF IRENCES}

(1) F. Najmabadi et al., "The ARIES-I Tokamak Reactor Study," Univ, of California Los Angles report UCLA-PPG-1323 (1991).

[2] F. Najmabadi et al., "The ARIES-III D. ${ }^{3} \mathrm{He}$ Tokamak Reactor Study," Univ. of Cal. Los Angles report UCLA-PPG-1384 (1992).

(3) F. Najnabadi et al., "The ARIES-II and -IV Second Stability Tbkamak Reactors," University of California Los Angles report UCLA-PPG-1461 (1992).

[4] C.G. Bathke and the ARIES Team, "A Comparison of SteadyState and Pulsed Tokamak Reactors," these proceed.

[5] K.A. Werley, "Reversed Field Pinch Ignition Requirements," Nuclear Fusion 31, 576 (1991).

[6] D.E. Post et al., "ITER Physics," International Thermonuclear Experimental Reactor doc. series, No. 21, IAEA (1991).

[7] P.I.H. Cooke, UKAEA Culham Lab., private commun. (1989).

[8] M. Greenwald et al., "A New Look at Density Limits in Tbkamaks," Nuclear Fusion 28 (1988) 2199. 

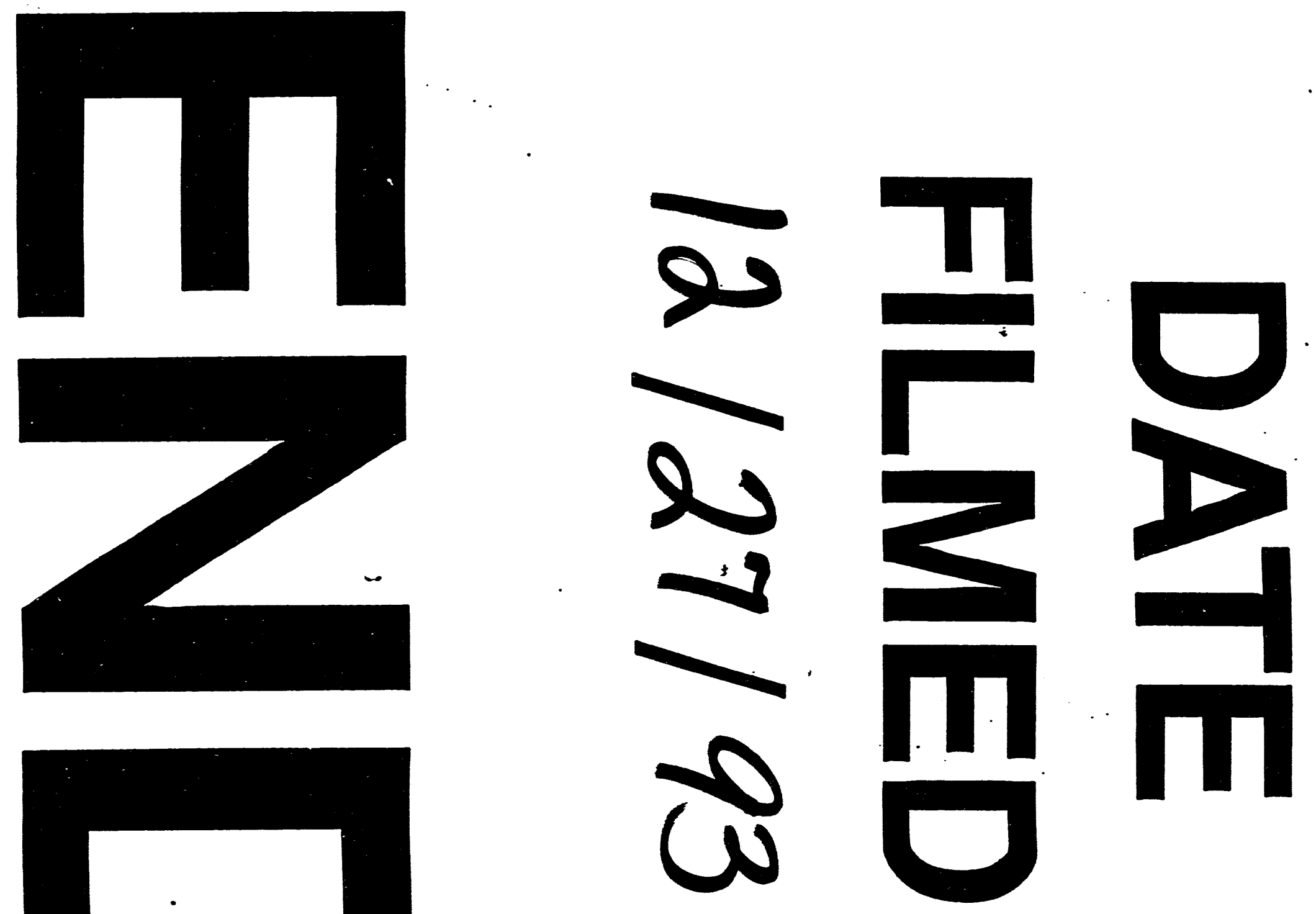
